\title{
DIFFERENTIABILITY OF THE METRIC PROJECTION IN FINITE-DIMENSIONAL EUCLIDEAN SPACE
}

\author{
EDGAR ASPLUND
}

Abstract. The metric projection on a closed subset of a finite-dimensional Euclidean space is almost everywhere differentiable.

The main purpose of this short note is to point out that the answer to a question by Kruskal [3] is implicit in a famous theorem of A. D. Alexandrov [1] (of which a new proof has recently been given by Rešetnjak [4]) which says that each continuous convex function on $\boldsymbol{R}^{n}$ is almost everywhere twice differentiable. For $n=1$, this reduces to Lebesgue's theorem about the differentiability almost everywhere of a monotone function.

Using Alexandrov's theorem one can prove the following theorem which contains the answer to Kruskal's question.

THEOREM. The metric projection on any closed subset of a finite-dimensional Euclidean space is almost everywhere differentiable.

Consider $\boldsymbol{R}^{n}$ as provided with the standard Euclidean norm. For a closed $K \subset \boldsymbol{R}^{n}$ and an element $x \in \boldsymbol{R}^{n}$ let $p(x)$ be the nearest point in $K$ to $x$. This may not be everywhere uniquely defined (as a matter of fact, this happens if and only if $K$ is convex) but we make a selection and call the function $p: \boldsymbol{R}^{n} \rightarrow K$ so defined the metric projection on $K$. In Asplund [2, p. 42 et seq.], it is shown that the convex function $f$, defined and continuous on all of $\boldsymbol{R}^{n}$ by

$$
f(x)=\sup \left\{(x, y)-\|y\|^{2} / 2 \mid y \in K\right\}=\|x\|^{2} / 2-\inf _{y \in K}\|x-y\|^{2} / 2,
$$

has $p(x)$ for a differential at all points $x$ where $f$ is once differentiable. Moreover, at those points where $f$ is not differentiable, $p(x)$ is an element of the subdifferential of $f$ at $x$. These are all easy facts, and the details of the calculations can be found in the paper [2]. An obvious calculation along the same lines then shows that our theorem here is a consequence of Alexandrov's.

Received by the editors March 18, 1971.

AMS (MOS) subject classifications (1969). Primary 5240.

Key words and phrases. Metric projection, differentiability.

(C) American Mathematical Society 1973 
The technique, used here and in [2], to represent a metric projection as the gradient of a convex function works only in Euclidean space. It would therefore be interesting to know for which finite-dimensional Banach spaces it is true that the metric projection on each closed subset is almost everywhere differentiable.

\section{REFERENCES}

1. A. D. Alexandrov, Almost everywhere existence of the second differential of a convex function and some properties of convex surfaces connected with it, Leningrad State Univ. Ann. Math. Ser. 6 (1939), 3-35. MR 2, 155.

2. E. Asplund, Fréchet differentiability of convex functions, Acta Math. 121 (1968), 31-47. MR 37 \#6754.

3. J. B. Kruskal, Two convex counterexamples: A discontinuous envelope function and a nondifferentiable nearest-point mapping, Proc. Amer. Math. Soc. 23 (1969), 697-703. MR 41 \#4385.

4. Ju. G. Rešetnjak, Generalized derivatives and differentiability almost everywhere, Mat. Sb. 75 (117) (1968), 323-334=Math. USSR Sb. 4 (1968), 293-302. MR 37 \#754.

The Institute for Optimization and Systems Theory, The Royal Institute of TeChNology, Stockholm 70, Sweden

Current address: Department of Mathematics, Odense University, DK-5000 Odense, Denmark 